\title{
PRICISE TARGET GEOLOCATION BASED ON INTEGERATION OF THERMAL VIDEO IMAGERY AND RTK GPS IN UAVS
}

\author{
H.R.Hosseinpoor, F.Samadzadegan, F. DadrasJavan* \\ Department of Geomatics Engineering, Faculty of Engineering, University of Tehran, Tehran, Iran - (hosseinpoor, samadz, \\ fdadrasjavan)@ut.ac.ir
}

Commission VI, WG VI/4

KEY WORDS: Thermal Video, RTK, Geolocation, Kalman Filter, Unmanned Aerial Vehicle

\begin{abstract}
:
There are an increasingly large number of uses for Unmanned Aerial Vehicles (UAVs) from surveillance, mapping and target geolocation. However, most of commercial UAVs are equipped with low-cost navigation sensors such as C/A code GPS and a lowcost IMU on board, allowing a positioning accuracy of 5 to 10 meters. This low accuracy which implicates that it cannot be used in applications that require high precision data on $\mathrm{cm}$-level. This paper presents a precise process for geolocation of ground targets based on thermal video imagery acquired by small UAV equipped with RTK GPS. The geolocation data is filtered using a linear Kalman filter, which provides a smoothed estimate of target location and target velocity. The accurate geo-locating of targets during image acquisition is conducted via traditional photogrammetric bundle adjustment equations using accurate exterior parameters achieved by on board IMU and RTK GPS sensors and Kalman filtering and interior orientation parameters of thermal camera from pre-flight laboratory calibration process.
\end{abstract}

\section{INTRODUCTION}

Small Unmanned Aerial Vehicles (UAVs) equipped with small, inexpensive on-board thermal cameras have attracted increased interest in recent. The advent of this new technology serves several applications such as rapid surveillance of an area by a search-and-rescue team, or helping aid-workers identify problem areas in need of immediate attention in a disaster torn area (Beard 2012, Madison 2008).

For these purposes, precise ground target localization from video imagery is crucially essential. Ivey (2005), Campbell. (2006), Redding (2006), Pachter (2007), Conte (2008), Quintero. (2010) Sharma. (2013), Shukla. (2014). Achieving accurate position deals great challenges when commercial small unmanned platforms are used. Since payload weight and size in small UAVs are limited, they can only carry very light sensors. Such sensors usually have poor performance which lowers the accuracy of localizing ground targets. Eling et al. (2014) Moreover, small and light active devices, such as radars and lasers, are not available to allow airborne applications on small platforms. So, the most common sensors used to geo-locate ground objects from a UAVs platform are passive video cameras due to advent of light and small video cameras. (Mallick 2007)

The detection of a ground target within the image frame can be performed manually, or automatically using image processing methods. Subsequently, the target coordinates can be calculated using the UAV position, attitude and the camera orientation relative to the UAV body. The UAV position is given by an onboard GPS receiver, while the attitude angles are computed from a navigation filter which integrates the inertial sensors (gyroscopes and accelerometers) and the GPS (Barton 2012). The problem of direct geo-locating of images with on-board GPS and IMU sensors is that the measurement of the UAV position, attitude and camera angles are usually affected by several error sources which lead to ground target localization errors. These errors can be addictive and result in ground resolution with up to tens of meters.

Traditional Global Positioning System (GPS) uses the timing differences between signals transmitted from satellites to a receiver which then digitally processes the data in order to find a location. This traditional method however, has an accuracy error of approximately $\sim 10 \mathrm{~m}$. In Real Time Kinematic GPS, there is a Base station module on the ground as well as a Rover. As long as the Rover and the Base maintain at least 5 satellites in common, there can be a more accurate locational prediction of the Rover by adjusting the corrections determined by the Base station. This RTK solution can provide centimetre grade accuracy of the position, and should cause a greater than 200 times increase in accuracy compared to traditional GPS. The major benefits are the extreme precision of the GPS unit for any application, with an option for real time tracking, it will be a crucial player in the future of UAV technology.

RTK-GPS is a differential GPS procedure that is based on carrier phase GNSS (Global Navigation Satellite System) observations and leads to relative positions between a master and a rover station with centimetre accuracy in real time. The challenge of developing such a system for micro- and minisized UAVs is to stay within the space and weight limitations of the platform. That is why only a few studies exist by now, dealing with the integration of a RTK-GPS system on micro- or mini-sized UAVs (Rieke 2011, Stempfhuber 2011).

\section{PROPSED METHOD}

This paper presents a real-time process for the identification and geolocation of ground targets based on thermal video imagery

\footnotetext{
* Corresponding author
} 
acquired by small UAV equipped with RTK GPS. The geolocation data is filtered using an extended Kalman filter, which provides a smoothed estimate of target location. RTK navigation provides high positioning performance in the range of a few centimetres; where main errors, including ionosphere and troposphere errors and satellite clock errors, among others are eliminated. For the proposed method, the time of image acquisition has to be aligned with the data stream of the camera exterior orientation. To use position data of a UAV in general within different use cases we have developed a software framework that overcomes several issues regarding the synchronization of different sensor data. It can be applied in different situations, especially for providing the trajectory of a UAV as a continuous time series. This mechanism is applicable and sufficient for scenarios where a high accurate position of the UAV is an inevitable necessity.

\subsection{Coordinate Frames and Conversion}

The geolocation algorithm uses a number of coordinate frames and considers transformations of 3 -vectors among coordinate frames. We assume that all coordinate frames are right-handed and orthogonal.

2.1.1 The inertial coordinate Frame $(I)$ is an earth-fixed coordinate system with its origin at the defined home location. As shown in Fig 2. This coordinate system is sometimes referred to as a north-east-down (NED) reference frame. It is common for north to be referred to as the inertial $x$ direction, east to be referred to as the inertial $y$ direction, and down to be referred to as the inertial $z$ direction. The transformation from vehicle frame to body frame is given by:

$$
R_{v}^{I}=\left(\begin{array}{c}
-x_{u a v} \\
-y_{u a v} \\
h_{u a v}
\end{array}\right)
$$

2.1.2 The vehicle frame $(v)$ is at the centre of mass of the MAV. However, the axes of $v$ are aligned with the axis of the inertial frame, in other word the $x$ direction points north, $y$ direction points east, and $z$ points toward the centre of the earth.

2.1.3 The body frame $(b)$ is vehicle-carried and is directly defined on the body of the flying vehicle. Its origin is the centre of mass, $x$ direction points out the nose of the airframe, $y$ direction points out the right wing, and $z$ direction points out the belly. The transformation from vehicle frame to body frame is given by

$$
\begin{aligned}
& R_{v}^{b}(\varphi, \theta, \psi)=R(\psi) R(\theta) R(\varphi) \\
& =\left[\begin{array}{ccc}
C_{\theta} C_{\psi} & C_{\theta} S_{\psi} & -S_{\theta} \\
S_{\varphi} S_{\theta} C_{\psi}-C_{\varphi} S_{\psi} & S_{\varphi} S_{\theta} S_{\psi}+C_{\varphi} C_{\psi} & C_{\theta} S_{\psi} \\
C_{\varphi} S_{\theta} C_{\psi}+S_{\varphi} S_{\psi} & C_{\varphi} S_{\theta} S_{\psi}-S_{\varphi} C_{\psi} & C_{\varphi} C_{\theta}
\end{array}\right]
\end{aligned}
$$

where, $C \varphi=\cos \varphi$ and $S \varphi=\sin \varphi$. The angles $\varphi, \theta$, and $\psi$ are commonly referred to as Euler angles. Euler angles are commonly used because they provide an intuitive means for representing the orientation of a body in three dimensions.

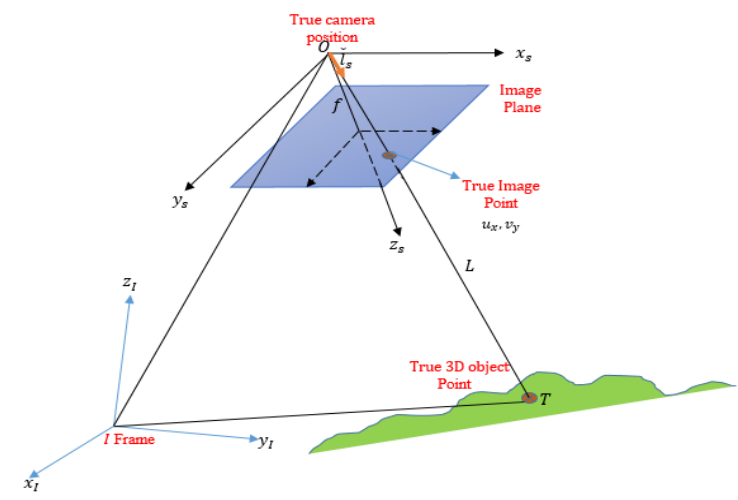

Figure 1: The orientation of the sensor frame ( $S$ frame) relative to the inertial coordinate frame ( $I$ frame).

The sensor frame $(S)$ - The origin of the s frame is at the optical centre of the camera with geodetic coordinates. The $\mathrm{z}$ axis is along the general downward direction along the optical axis. The $\mathrm{x}$ axis is to the right hand side of the image .The $\mathrm{y}$ axis completes the right-handed coordinate frame. The image points and normalized image points are expressed in the $\mathrm{S}$ frame $\left(u_{x}, v_{x}\right.$ f). Using similar triangles in Fig. 1:

$$
\begin{aligned}
F & =\sqrt{v_{x}{ }^{2}+u_{y}{ }^{2}+f^{2}} \\
l^{s} & =\frac{L}{F}\left(\begin{array}{l}
u_{x} \\
v_{x} \\
f
\end{array}\right)
\end{aligned}
$$

where $l=$ the vector to the object of interest and $\mathrm{L}=\|l\|$.

Note that $l^{s}$ cannot be determined strictly from camera data since $\mathrm{L}$ is unknown. However, we can determine the unit direction vector to the target as:

$$
\begin{aligned}
& \frac{l^{s}}{L}=\frac{1}{F}\left(\begin{array}{l}
u_{x} \\
v_{x} \\
f
\end{array}\right)=\frac{1}{\sqrt{v_{x}{ }^{2}+u_{y}{ }^{2}+f^{2}}}\left(\begin{array}{l}
u_{x} \\
v_{x} \\
f
\end{array}\right) \\
& \check{l}^{s}=\frac{l_{s}}{L}
\end{aligned}
$$

\subsection{Geolocation Algorithm}

This section presents a method for determining the location of objects in world/inertial coordinates using a video camera on board a fixed-wing UAV. From the Fig. 1 we have the relationship. (Beard 2012).

$$
\begin{aligned}
& P_{o b j}^{I}=P_{u a v}^{I}+R_{v}^{I} R_{b}^{v} R_{s}^{b} l^{s} \\
& =P_{u a v}^{I}+L\left(R_{v}^{I} R_{b}^{v} R_{s}^{b} \breve{l}^{s}\right) \\
& P_{u a v}^{I}=\left(\mathrm{p}_{\mathrm{n}}, \mathrm{p}_{\mathrm{e}}, \mathrm{p}_{\mathrm{d}}\right)^{T}
\end{aligned}
$$

The only element on the right-hand side of equation 8 , which is unknown is $L$. Therefore, solving the geolocation problem reduces to the problem of estimating the range to the target $L$. If digital elevation model is not available, simple strategy for estimating $L$ is to assume a flat-earth model. The geometry of the situation where $h=-p_{d}$ is the height-above-ground, and $\lambda$ is the angle between $l$ and direction $k^{i}$ axis. It is clear: 


$$
\begin{gathered}
\cos \lambda=k^{i} \cdot \breve{l}^{i}=k^{i} \cdot R_{v}^{I} R_{b}^{v} R_{s}^{b} \breve{l}^{s} \\
L=\frac{h}{k^{i} \cdot R_{v}^{I} R_{b}^{v} R_{s}^{b} \breve{l}^{s}}
\end{gathered}
$$

The Geolocation estimation is given by combining equation 8 and 11 as:

$$
P_{o b j}^{I}=P_{u a v}^{I}+h \frac{R_{v}^{I} R_{b}^{v} R_{s}^{b} \breve{l}^{s}}{k^{i} \cdot R_{v}^{I} R_{b}^{v} R_{s}^{b} \breve{l}^{s}}
$$

2.2.1 Geolocation using extended Kalman filter: The geolocation estimate in equation 12 provides a one-shot estimate of the target location. Unfortunately, this equation is highly sensitive to measurement errors, especially attitude estimation errors of the airframe. In this section we will describe the use of the extended Kalman filter (EKF) to solve the geolocation problem. If we assume the object is stationary the state vector of dynamic system is given by:

$$
X_{k}=\left[\mathrm{t}_{\mathrm{n}}, \mathrm{t}_{\mathrm{e}}, \mathrm{L}\right]
$$

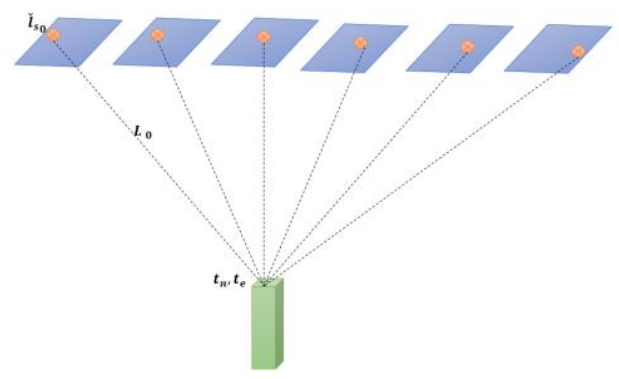

Figure 2: Point location in sequence of frames

$$
\begin{gathered}
L=\left\|P_{o b j}-P_{u a v}\right\| \\
\dot{L}=\frac{d}{d t} \sqrt{\left(P_{o b j}-P_{u a v}\right)^{T}\left(P_{o b j}-P_{u a v}\right)} \\
=\frac{\left(P_{o b j}-P_{u a v}\right)^{T}\left(\dot{P}_{o b j}-\dot{P}_{u a v}\right)}{L} \\
=-\frac{\left(P_{o b j}-P_{u a v}\right)^{T} \dot{P}_{u a v}}{L} \\
\dot{P}_{u a v}=\left(\begin{array}{c}
\hat{v_{g}} \cos \hat{\chi} \\
\hat{v_{g}} \sin \hat{\chi} \\
0
\end{array}\right)
\end{gathered}
$$

where $t_{n}, t_{n}=$ north and east position coordinate of target, $\dot{P}_{\text {uav }}$ $=$ the UAV velocity, $\hat{v}_{g}$ and $\hat{\chi}=\mathrm{UAV}$ ground speed and course angle.

The prediction step for filter corresponding to the target is given by:

$$
\begin{aligned}
& \bar{X}_{k+1}=\hat{X}_{k}+\Delta t \times f\left(\hat{X}_{k}\right) \\
& \bar{P}_{k+1}=F_{k} P_{k} F_{k}{ }^{T}+Q
\end{aligned}
$$

Where the $P_{k}$ is the state covariance matrix for the target at time step $\mathrm{k}, \Delta \mathrm{t}$ is the sampling period and $F_{k}$ is the system Jacobian matrix.

$$
\begin{aligned}
& F_{k}=\frac{\partial f(\mathrm{X})}{\partial X} \\
& =\left(\begin{array}{cc}
0 & 0 \\
-\frac{\hat{\dot{P}}_{u a v}{ }^{T}}{\hat{L}} & \frac{\left(\hat{P}_{o b j}-\hat{P}_{u a v}\right)^{T} \hat{\dot{P}}_{u a v}}{\hat{L}^{2}}
\end{array}\right)
\end{aligned}
$$

Now for measurement model we use equation 8, rearranging this equation we get

$$
\begin{aligned}
& P_{u a v}^{I}=P_{o b j}^{I}-L\left(R_{v}^{I} R_{b}^{v} R_{s}^{b} \breve{l}^{s}\right) \\
& P_{u a v}^{I}=h\left(\mathrm{X}_{\mathrm{k}}\right)+v_{k}
\end{aligned}
$$

The position of UAV is measured by GPS, will be used as the measurement equation, assuming that GPS noise is zero-mean Gaussian. However the measurement model update for this system is given by:

$$
\begin{aligned}
& r_{k}=h\left(\mathrm{X}_{\mathrm{k}}\right)+h\left(\overline{\mathrm{X}}_{\mathrm{k}}^{-}\right) \\
& S_{k}=H\left(\overline{\mathrm{X}}_{\mathrm{k}}^{-}\right) \mathrm{P}_{k}^{-} H\left(\overline{\mathrm{X}}_{\mathrm{k}}^{-}\right)^{T}+R_{k} \\
& K_{k}=\mathrm{P}_{k}^{-} H\left(\overline{\mathrm{X}}_{\mathrm{k}}^{-}\right)^{T} S_{k}{ }^{-1} \\
& \overline{\mathrm{X}}_{\mathrm{k}}=\overline{\mathrm{X}}_{\mathrm{k}}^{-}+K_{k} r_{k} \\
& \overline{\mathrm{X}}_{\mathrm{k}}=\mathrm{P}_{k}^{-}-K_{k} S_{k} K_{k}{ }^{T}
\end{aligned}
$$

where $H$ is the measurement Jacobian given by :

$$
\begin{aligned}
& \mathrm{H}\left(\mathrm{X}_{\mathrm{k}}\right)=\frac{\partial h(\mathrm{X})}{\partial X} \\
& =\left(\begin{array}{ll}
I & R_{v}^{I} R_{b}^{v} R_{s}^{b} \breve{l}^{s}
\end{array}\right)
\end{aligned}
$$

\section{EXPERIMENTAL RESULTS}

We used a commercial multirotor UAV platform for evaluation of proposed method. The custom design of the multirotor allows us to mount all the necessary devices needed to perform target geo-location. The platform is equipped with small thermal video camera and with appropriate sensors and autopilot to perform stabilized and autonomous flights. The autopilot unit includes MEMS gyroscopes and accelerometers, a 3-axis magnetic sensor, a barometric pressure sensor and a single frequency lowcost GPS receiver and a low-cost, high-performance GPS receiver with Real Time Kinematics (RTK) functionality for decimetre level positioning accuracy. A base station and a rover station can cover a service area of about 20 square kilometers. The data transition is in real time using a wireless VHF modem. The cooperation of these navigation components allows horizontal and vertical stabilized positioning of the whole system as well as the position hold, return to the launch site or other various features including the mission flying.

The thermal video acquired by TC688 camera which is mount on the platform. The specifications of camera are presented in Table 1.

Table 1: Thermal camera specification

\begin{tabular}{c|c}
\hline Parameters & Values \\
\hline Detector type & Uncooled FPA \\
\hline Pixel pitch & $17 \mu \mathrm{m}$ \\
\hline IR resolution & $640 \times 480$ \\
\hline Focal length & 25 \\
\hline Frequency & $50 \mathrm{~Hz}$ \\
\hline Sensitivity & $<65 \mathrm{mk} @ \mathrm{f} / 1.0$ \\
\hline
\end{tabular}




\subsection{Geometric Calibration}

Geometric calibration of thermal camera with planar pattern is performed in laboratory. The test field consists of a grid of 28 regularly sized circle with 4 coded targets cut out of a thin nonconductor material. The size of mask pattern is $44 \times 24 \mathrm{~cm}^{2}$, diameter of squares are $20 \mathrm{~mm}$ which spaced with $50 \mathrm{~mm}$ separation. Fig. 3 shows the thermal imagery acquired from mask based calibration pattern. The pattern is held in front of a powered computer monitor.

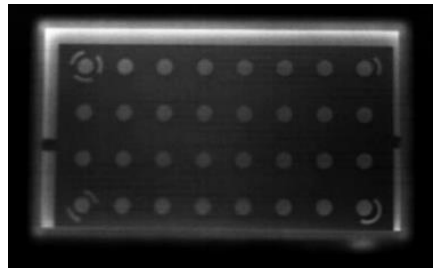

Figure 3. Thermal imagery for calibration

By starching, thermal image contrast are increased. Then subpixel accurate positions of the centre of each circle is defined.

Self-calibration is performed to compute camera calibration parameters. The results of camera calibration are presented in Table 2.

Table 2; Thermal camera specification

\begin{tabular}{c|c|c}
\hline Parameters & Values & SD \\
\hline $\boldsymbol{C}$ & 25.8655 & 0.525 \\
\hline $\boldsymbol{x}_{\boldsymbol{p}}$ & 0.0085 & 0.235 \\
\hline $\boldsymbol{y}_{\boldsymbol{p}}$ & -0.9934 & 0.235 \\
\hline $\boldsymbol{K}_{\boldsymbol{1}}$ & $-5.54162 \mathrm{e}-004$ & $1.1728 \mathrm{e}-003$ \\
\hline $\boldsymbol{K}_{\boldsymbol{2}}$ & $1.46497 \mathrm{e}-004$ & $1.17294 \mathrm{e}-004$ \\
\hline $\boldsymbol{K}_{\boldsymbol{3}}$ & $-8.38681 \mathrm{e}-004$ & $1.0917 \mathrm{e}-005$ \\
\hline $\boldsymbol{P}_{\boldsymbol{1}}$ & $-7.2475 \mathrm{e}-004$ & $3.984 \mathrm{e}-004$ \\
\hline $\boldsymbol{P}_{\boldsymbol{2}}$ & $-6.5166 \mathrm{e}-004$ & $6.079 \mathrm{e}-004$ \\
\hline $\boldsymbol{B}_{\boldsymbol{1}}$ & $1.1377 \mathrm{e}-002$ & $2.241 \mathrm{e}-003$ \\
\hline $\boldsymbol{B}_{\boldsymbol{2}}$ & $1.3384 \mathrm{e}-002$ & $3.548 \mathrm{e}-003$ \\
\hline
\end{tabular}

\subsection{Flight Data}

The altitude of the flight was $100 \mathrm{~m}$ above ground, results in a ground resolution of about $7 \mathrm{~cm}$. The flight lines were planned for the images to have an overlap of $60 \%$ between the image stripes. According to the frame rate of the camera, flight velocity, flight height and the $80 \%$ overlap, the frames are extracted. Fig. 4 demonstrates some extracted frames.

For accuracy assessment of proposed method, 3D dense DSM of the area which is generated from visible aerial imagery is applied. Deviation of extracted coordinates for each object from proposed algorithm with respect to DSM based coordinate considered as the measure of accuracy.
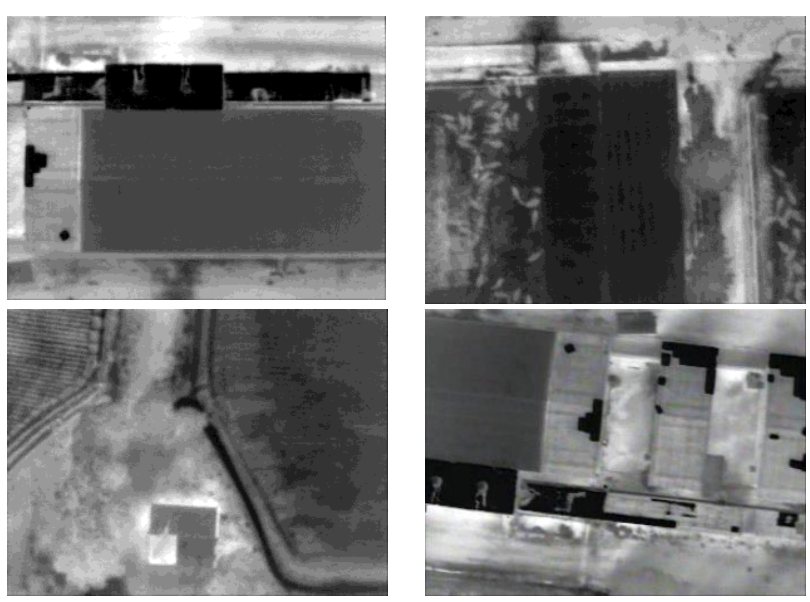

Figure 4: Frame extracted from thermal video
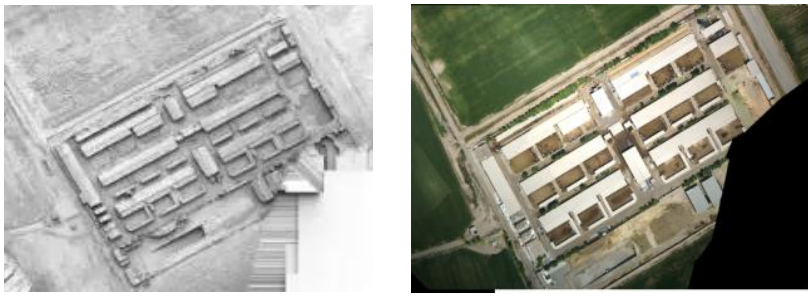

Figure 5: 3D dense reference data

\subsection{Object Tracking}

Video sequence Object tracking consists of determining the image coordinates of an object of interest in consecutive video frames. One possible approach is to use the mean shift algorithm to localize the target object. The mean shift algorithm is a robust statistical method which finds local maxima in any probability distribution. It works with a search window that is positioned over a section of the distribution. Within this search window the maximum can be determined by a simple average computation. Then the search window is moved to the position of this maximum and the average computation is repeated again. This procedure is repeated until the mean shift algorithm finds a local maximum and converges. To apply the mean shift algorithm in the field of object tracking it is necessary to represent the data of video frames as a probability distribution. Every pixel in a frame gets a probability value $P(u, v)$, depending on its color. $P$ is a value which indicates how likely it is that the related pixel belongs to the target object. Using this probability values a frame can be represented as a 2D probability distribution and the mean shift algorithm can be applied. The output of this phase is the coordinates of the target in the sensor frame (Fig. 6). 

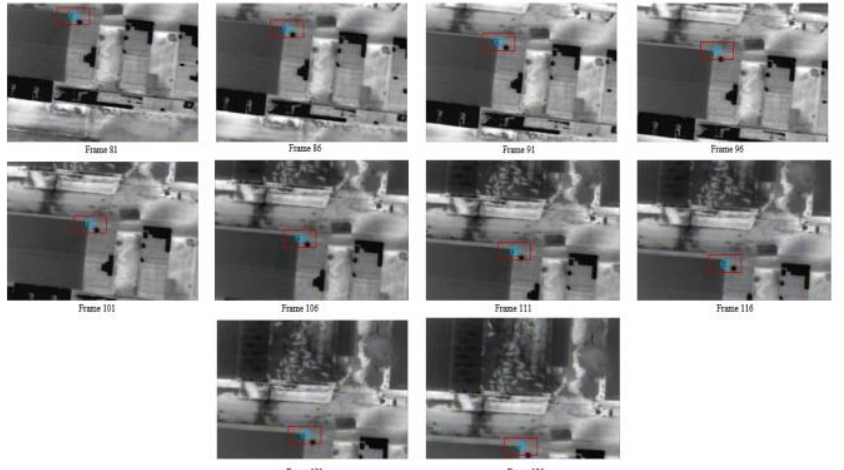

Figure 6: Target tracked in the sequence of successive frames

\subsection{Result}

In Fig. 7, the operator selected seven interest targets by clicking on the first frame. Using mean-shift tracking algorithm the target tracked in subsequent frames and coordinate of the centre of the target computed from geolocation algorithm in sensor frame Fig. 8. First we use intersection of tow rays from stereo frame to compute location of selected target. Then, the proposed method to track and estimate target position applied with an extended Kalman filter. Coordinates of targets are extracted and compared to those extracted from reference data. Extracted coordinates for presented target in Fig 6 are presented in table 3.

Table 3: Standard deviation in geolocation for target 1

\begin{tabular}{c|c|c|c|c}
\hline method & Northing & Easting & DN(m) & DE(m) \\
\hline SPP GPS & 35.430865 & 51.637307 & 20.6 & 18.3 \\
\hline RTK GPS & 35.4307837 & 51.6373359 & 1.1 & 1.08 \\
\hline $\begin{array}{l}\text { RTK GPS } \\
\text { with EKF }\end{array}$ & 35.4307737 & 51.6372381 & 0.45 & 0.39 \\
\hline
\end{tabular}

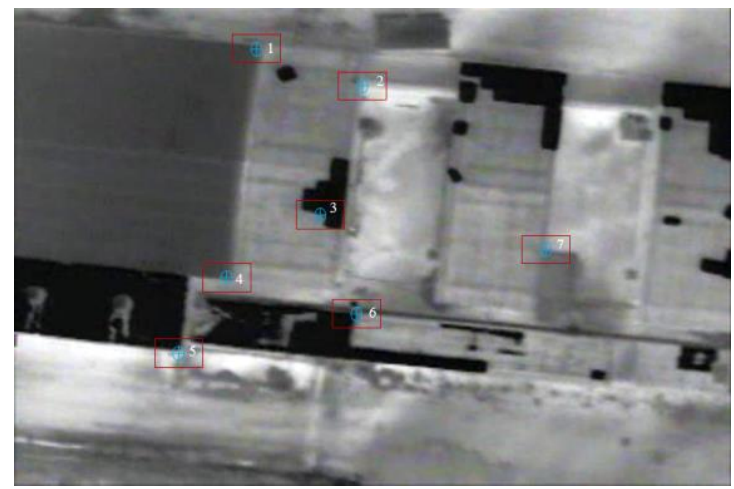

Figure 7: Targets extracted for tracking and geolocation in first frame

The coordinates for all extracted points are determined based on developed process. Mean standard deviation of coordinates with respect to reference data for all target extracted and shown in Table 4 .

Table 4: Mean Std achieved for all extracted targets

\begin{tabular}{c|c}
\hline method & Mean Std \\
\hline SPP GPS & 19.5 \\
\hline RTK GPS & 1.1 \\
\hline RTK GPS with EKF & 0.45 \\
\hline
\end{tabular}

\section{CONCLUSION}

To estimate the 3D coordinates of ground target, target position is computed by intersecting the ray starting from the camera centre and passing through the target pixel location in the image plane with the ground. These parameters are used in the traditional UAV geolocation methods. The resulting accuracies of such sensor combinations are $2-10 \mathrm{~m}$ for the positions and 0.5-5 deg for the attitudes. While these accuracies are sufficient for a rough navigation of a UAV, they are mostly insufficient for a direct geo-locating of targets in the UAV video Imagery.

In this paper a real-time process for the identification and geolocation of ground targets based on video imagery acquired by small UAV equipped with RTK GPS is proposed. Results are compared with positioning accuracy acquired with codebased ordinary GPS instead of RTK which shows improvement of accuracy to decimetres. Therefore, it can be concluded that the proposed platform is relatively safe and inexpensive for collecting critical spatial information for urgent response such as disaster relief and assessment applications where GCPs are not available.

\section{REFRENCES}

Barton, J. D. 2012. Fundamentals of small unmanned aircraft flight. Johns Hopkins APL technical digest 31(2): 132-149.

Beard, R. W., 2012. Small unmanned aircraft: Theory and practice, Princeton University Press.

Campbell, M. E., 2006. A vision based geolocation tracking system for UAVs. Proceedings of the AIAA Guidance, Navigation, and Control Conference and Exhibit.

Conte, G., 2008. High accuracy ground target geo-location using autonomous micro aerial vehicle platforms. Proceedings of the AIAA-08 Guidance, Navigation, and Control Conference.

Eling, C., 2014. A presice direct georefrncing system for UAVS., Proceedings of the Workshop on UAV-basaed Remote Sensing Methods for Monitoring Vegetation.Kölner Geographische Arbeiten, 94. Köln:33-41

Ivey, G.F., 2005. Investigation of methods for target state estimation using vision sensors., AIAA Guidance, Navigation, and Control Conference and Exhibit, San Francisco, CA, Aug.

Mallick, M., 2007. Geolocation using video sensor measurements. Information Fusion, 2007 10th International Conference on, IEEE.

Pachter, M., 2007. Vision-based target geolocation using feature tracking. AIAA Guidance, Navigation and Control Conference and Exhibit, Hilton Head, South Carolina.

Quintero, S. A., 2010. Optimal UAV coordination for target tracking using dynamic programming. Decision and Control (CDC), 2010 49th IEEE Conference on, IEEE.

Rieke, M., 2011. High-precision positioning and real-time data processing of UAV-systems. International Archives of Photogrammetry, Remote Sensing and Spatial Information Sciences 38: 1-C22. 
Redding, J. D., 2006. Vision-based target localization from a fixed-wing miniature air vehicle. American Control Conference, 2006, IEEE.

Sharma, R., 2013. Cooperative Sensor Resource Management for Multi Target Geolocalization using Small Fixed-wing Unmanned Aerial Vehicles. AIAA Guidance, Navigation, and Control.

Shukla, P., 2014. Automatic geolocation of targets tracked by aerial imaging platforms using satellite imagery. ISPRSInternational Archives of the Photogrammetry, Remote Sensing and Spatial Information Sciences 1: 1213-1220.

Stempfhuber., 2011. A precise, low-cost RTK GNSS system for UAV applications. International Archives of Photogrammetry, Remote Sensing and Spatial Information Science 38: 1-C22.

Madison, R., 2008. Target geolocation from a small unmanned aircraft system. Aerospace Conference, 2008 IEEE, IEEE. 